\title{
Density-matrix functionals from Green's functions
}

\author{
Peter E. Blöchl, ${ }^{1,2, *}$ Thomas Pruschke ${ }^{3}$ and Michael Potthoff ${ }^{4}$ \\ ${ }^{1}$ Clausthal University of Technology, Institute for Theoretical Physics, \\ Leibnizstr.10, D-38678 Clausthal-Zellerfeld, Germany \\ ${ }^{2}$ Georg-August Universität Göttingen, Institute for Materials Physics, \\ Friedrich-Hund-Platz 1, D-37077 Göttingen, Germany \\ ${ }^{3}$ Georg-August Universität Göttingen, Institute for Theoretical Physics, \\ Friedrich-Hund-Platz 1, D-37077 Göttingen, Germany \\ ${ }^{4}$ I. Institute for Theoretical Physics, University of Hamburg, Jungiusstr. 9, D-20355 Hamburg, Germany
}

(Dated: November 21, 2013)

\begin{abstract}
The exact reduced density-matrix functional is derived from the Luttinger-Ward functional of the single-particle Green's function. Thereby, a formal link is provided between diagrammatic manybody approaches using Green's functions on the one hand and theories based on many-body wave functions on the other. This link can be used to explicitly construct approximations for the densitymatrix functional that are equivalent to standard diagrammatic re-summation techniques and to non-perturbative dynamical mean-field theory in particular. Contrary to functionals of the Green'sfunction, the exact density-matrix functional is convex and thus provides a true minimum principle which facilitates the calculation of the grand potential and derived equilibrium properties. The benefits of the proposed Green's-function-based density-matrix functional theory for geometrical structure optimization of strongly correlated materials are discussed.
\end{abstract}

PACS numbers: 71.10.-w,71.15.Nc,71.15.Mb

\section{INTRODUCTION}

Consistent and reliable approximations for extended systems of correlated electrons are ideally generated by making use of general variational principles. Two fundamentally different concepts have been pursued in the past, namely wave-function-based and Green's-functionbased methods.

(i) Wave-function-based methods make use of the Ritz variational principle, which relies on the optimization of the many-body ground-state wave function or, at finite temperatures, the statistical operator. Densityfunctional theory (DFT) ${ }^{1-4}$ and reduced density-matrix functional theory (rDMFT) $)^{3,5-10}$ are based on the optimization of the electron density or the reduced oneparticle density matrix, respectively, and the underlying variational principles derive from the Ritz principle. The Ritz principle actually represents a minimum principle, i.e., the ground-state energy or, at finite $T,{ }^{11}$ the grand potential is at a minimum for the exact ("physical") wave function or density operator. Analogously, within DFT and rDMFT, the physical electron density or the physical one-particle reduced density matrix, respectively, minimize the corresponding functional. Within condensedmatter electronic-structure theory, approximations derived from those wave-function-based approaches, e.g. the local-density approximation ${ }^{1,2}$ within DFT, are usually employed to study weakly or moderately correlated materials.

(ii) Strongly correlated electron systems are often studied with Green's-function techniques. The one-electron Green's function is closely related to the spectrum of one-particle excitations accessible to photoemission spectroscopy. It can be obtained from a dynamical varia- tional principle, ${ }^{12-14}$ named after Kadanoff and Baym, which expresses the grand potential as a functional of the frequency-dependent Green's function or as a functional of the Green's function and the self energy. This and related variational principles ${ }^{15-17}$ are based on the Luttinger-Ward functional. ${ }^{12,18}$ Dynamical variational principles represent stationary principles, i.e., the grand potential is stationary - but not necessarily extremal - at the physical Green's function and the physical self energy. Despite some attempts ${ }^{19,20}$, there is, with the exception of certain highly symmetric cases ${ }^{21}$, no known general dynamical functional which is convex. Self-energy functional theory ${ }^{16}$ has explicitly shown that evaluating a dynamical functional at trial self-energies away from a stationary point may yield a grand potential smaller than the physical one.

The reason for this deficiency of Green's-functionbased theories lies in the fact that there is no general relation by which dynamical functionals of the Green's function or self energy can be transformed into functionals of the many-body wave function while preserving the variational properties.

In this paper, we derive the density-matrix functional from the Luttinger-Ward functional and thus provide a link between wave-function-based and Green's-functionbased approaches. The density-matrix functional for a given one-particle density matrix is obtained as the value of a functional of the one-particle Green's function and the self energy at its stationary point. The densitymatrix functional obtained in this way, inherits a true minimum principle from its wave-function-based origin.

The conceptual benefit from this construction is that well-known diagrammatic approximations within manybody perturbation theory will be "translated" into cor- 
responding approximations within the framework of rDMFT. Standard conserving approximations ${ }^{13,14}$ include the Hartree-Fock (HF) approximation and weakcoupling approximations, such as the random-phase approximation (RPA), $G W$ or the fluctuation-exchange (FLEX) approximation. However, also the nonperturbative dynamical mean-field theory can be obtained variationally using the one-particle density matrix as the basic variable.

The stationary property of dynamical functionals has been exploited earlier, namely to obtain improved approximations for the total energy using an approximate Green's function or self energy. ${ }^{22}{ }^{-25}$. With a minimum principle at hand, one is in a position to compute strict upper bounds to the total energy additionally and thereby to compare the quality of different approximate Green's functions.

Convexity of the density-matrix functional is also very helpful for numerical purposes: ${ }^{26}$ There are a number of efficient general techniques for finding an extremum of a multidimensional function. For the search of stationary points, including saddle points, on the other hand, one generally has to resort to less efficient multidimensional root finding.

Furthermore, geometry optimization is greatly facilitated if there is a minimum principle available for the total energy given in terms of electronic degrees of freedom. This is of interest for materials with strong electronic correlations, which often exhibit a strong mutual dependence between the electronic equilibrium and the geometrical structure. In particular, electronically driven phase transitions are often accompanied by structural relaxations. Here, the prototypical method that we have in mind is the fictitious-Lagrangian approach of $a b i n i$ tio molecular dynamics ${ }^{27}$ which performs the electronic and geometric optimization simultaneously. One big advantage of this method is that the calculations of forces do not require the inclusion of the linear response of the electronic structure to a virtual displacement, even if the electronic structure is not at its ground state. Ab initio molecular dynamics is not only useful for structure optimization, but also for the study of atomic motion either for investigating the dynamics or, exploiting the ergodic principle, the ensemble properties at finite temperature. However, ab initio molecular dynamics depends on a true minimum principle for the electronic degrees of freedom.

Finally, a further benefit of constructing densitymatrix functionals using Green's function techniques consists in the possibility to find explicit expressions for approximate density-matrix functionals that are consistent with specific diagrammatic approximations or have a comparable quality or reliability. This would allow us to bypass the complexity of a diagrammatic many-body calculation, i.e., to work with the much simpler density matrix rather than with Green's functions or self-energies. For geometry optimization, but also in other contexts, this would be extremely helpful. Although dynamical (frequency-dependent) quantities will be disregarded in such an approach, one would still have full access to spectral properties in the end. Namely, the complexity of calculating spectral properties can be postponed to the converged state. Quite generally, we will show how to obtain a spectral function from a converged density matrix consistent with the approximation underlying the density-matrix calculation.

The purpose of the present paper is to present the theoretical setup. Sec. II presents the notation and introduces the basics of density-matrix functional theory as well as of the Luttinger-Ward functional. In Sec. III, we show how an intrinsic symmetry of the Kadanoff-Baym functional leads to the definition of the density-matrix functional in terms of a stationary functional of Green's functions and self energies. In Sec. IV, we discuss some common approximations and in Sec. V, we discuss the implications on force calculations and spectral properties.

\section{VARIATIONAL PRINCIPLES WITH WAVE FUNCTIONS AND GREEN'S FUNCTIONS}

\section{A. Density-matrix functional approach}

The grand potential for a many-particle system has the form

$$
\Omega_{\beta, \mu}(\hat{h}+\hat{W})=-\frac{1}{\beta} \ln \left[\operatorname{Tr}\left\{\mathrm{e}^{-\beta(\hat{h}+\hat{W}-\mu \hat{N})}\right\}\right],
$$

where $\beta=1 /\left(k_{B} T\right)$, with the Boltzmann constant $k_{B}$ and the temperature $T$. The trace is performed over the Fock space of the electron gas. Furthermore,

$$
\hat{h}=\sum_{a, b} h_{a, b} \hat{c}_{a}^{\dagger} \hat{c}_{b}
$$

is the non-interacting part of the Hamiltonian expressed by creation and annihilation operators in an orthonormal one-particle basisset,

$$
\hat{W}=\frac{1}{2} \sum_{a, b, c, d} U_{a, b, d, c} \hat{c}_{a}^{\dagger} \hat{c}_{b}^{\dagger} \hat{c}_{c} \hat{c}_{d}
$$

is the electron-electron interaction, and $\hat{N}$ is the particle number operator

$$
\hat{N}=\sum_{a} \hat{c}_{a}^{\dagger} \hat{c}_{a}
$$

In the following, bold-faced symbols are matrices in the one-particle Hilbert space. Derivatives with respect to matrices are interpreted in the form $\left(\frac{\partial Y}{\partial A}\right)_{a, b}=\frac{\partial Y}{\partial A_{b, a}}$.

The wave-function approach to the grand-canonical ensemble is to minimize the grand potential, expressed as a functional of (fermionic) many-particle wave functions 
$\left|\Phi_{j}\right\rangle$ in Fock space and their probabilities $P_{j}$ :

$$
\begin{aligned}
\Omega_{\beta, \mu}(\hat{h}+\hat{W})= & \min _{P_{j} \geq 0,\left|\Phi_{j}\right\rangle \Lambda, \lambda} \operatorname{stat}_{\Lambda, \lambda}\left\{\frac{1}{\beta} \sum_{j} P_{j} \ln \left[P_{j}\right]\right. \\
& +\sum_{j} P_{j}\left\langle\Phi_{j}|\hat{h}+\hat{W}-\mu \hat{N}| \Phi_{j}\right\rangle \\
& -\sum_{i, j} \Lambda_{i, j}\left(\left\langle\Phi_{j} \mid \Phi_{i}\right\rangle-\delta_{j, i}\right) \\
& \left.-\lambda\left(\sum_{j} P_{j}-1\right)\right\} .
\end{aligned}
$$

With $\mu$, we denote the chemical potential of the electrons. We introduce the symbol "stat" to denote a stationary condition, which may be an extremum or a saddle point.

The constraints of orthonormal wave functions and the sum rule for the probabilities are enforced using Lagrange multipliers $\Lambda_{i, j}$ and $\lambda$. The probabilities must be positive. In practice, this requirement is enforced by expressing the probabilities as squares of real-valued variables.

Following the constrained-search method of Levy ${ }^{3}$, the minimization with respect to all many-particle wave functions can be divided into two steps. (1) For each oneparticle reduced density matrix $\boldsymbol{\rho}$, we collect all fermionic many-particle ensembles, consisting of sets of antisymmetric many-particle wave functions $\left|\Phi_{j}\right\rangle$ in Fock space and their probabilities $P_{j}$, which yield this density matrix via

$$
\rho_{a, b}:=\sum_{j} P_{j}\left\langle\Phi_{j}\left|\hat{c}_{b}^{\dagger} \hat{c}_{a}\right| \Phi_{j}\right\rangle
$$

Then, one identifies the minimum of the grand potential for this subset. (2) In the second step, we minimize the grand potential with respect to the one-particle density matrix.

The first part in this two-step minimization defines a functional of the one-particle reduced density matrix. This density-matrix functional is then used to determine the minimum in the second part, which is performed without referring to many-particle wave functions: All relevant information from the many-particle wave functions has been encoded in the density-matrix functional.

To obtain a density-matrix functional, one splits the grand potential into contributions that can be expressed by the one-particle density matrix alone and terms that can only be obtained from the many-particle wave function. Thus, we obtain

$$
\Omega_{\beta, \mu}(\hat{h}+\hat{W})=\min _{\boldsymbol{\rho}}\left\{\operatorname{Tr}[\boldsymbol{\rho}(\boldsymbol{h}-\mu \mathbf{1})]+F_{\beta}^{\hat{W}}[\boldsymbol{\rho}]\right\}
$$

with

$$
\begin{aligned}
F_{\beta}^{\hat{W}}[\boldsymbol{\rho}] & =\min _{P_{j} \geq 0,\left|\Phi_{j}\right\rangle} \operatorname{stat}_{\boldsymbol{h}^{\prime}, \boldsymbol{\Lambda}, \lambda}\left\{\sum_{j} P_{j}\left\langle\Phi_{j}|\hat{W}| \Phi_{j}\right\rangle\right. \\
& +\frac{1}{\beta} \sum_{j} P_{j} \ln \left[P_{j}\right] \\
& -\sum_{i, j} \Lambda_{i, j}\left(\left\langle\Phi_{j} \mid \Phi_{i}\right\rangle-\delta_{j, i}\right)-\lambda\left(\sum_{j} P_{j}-1\right) \\
& \left.+\sum_{a, b} h_{a, b}^{\prime}\left(\sum_{j} P_{j}\left\langle\Phi_{j}\left|\hat{c}_{a}^{\dagger} \hat{c}_{b}\right| \Phi_{j}\right\rangle-\rho_{b, a}\right)\right\} .
\end{aligned}
$$

The density-matrix functional $F_{\beta}^{\hat{W}}$ is the minimum of the interaction energy and the entropy term. Note, that the density-matrix functional does not vanish in the noninteracting limit but that it contributes at finite temperature the entropy term

$$
F_{\beta}^{\hat{0}}[\boldsymbol{\rho}]=\frac{1}{\beta} \operatorname{Tr}[\boldsymbol{\rho} \ln (\boldsymbol{\rho})-(\mathbf{1}-\boldsymbol{\rho}) \ln (\mathbf{1}-\boldsymbol{\rho})]
$$

of the non-interacting electron gas.

An important property of the density-matrix functional is its universality: The density-matrix functional is independent of the one-particle Hamiltonian, because the latter, i.e. $\boldsymbol{h}^{\prime}$ enters only as a Lagrange multiplier.

As shown in Appendix A, the density-matrix functional, defined as constrained search over ensembles, is convex.

The density matrix can be diagonalized in the oneparticle Hilbert space,

$$
\sum_{b} \rho_{a, b} \psi_{b, n}=\psi_{a, n} f_{n}
$$

which produces the so-called natural orbitals ${ }^{28}\left|\psi_{n}\right\rangle=$ $\sum_{a}\left|\chi_{a}\right\rangle \psi_{a, n}$ from the one-particle basisstates $\left|\chi_{a}\right\rangle$ as eigenstates of the density matrix and the occupations $f_{n}$ as its eigenvalues. The natural orbitals are orthonormal one-particle states, such that $\left\langle\psi_{m} \mid \psi_{n}\right\rangle=\delta_{m, n}$.

The density matrix must obey the so-called $N$ representability property, that is it must be a matrix that can be obtained from an ensemble of (fermionic) manyparticle wave functions. Coleman ${ }^{6}$ has shown that this condition is identical to the requirement that the density matrix be Hermitian and its eigenvalues, the occupations, lie between zero and one.

The grand potential can be expressed more conveniently by the natural orbitals and their occupations rather than directly by the one-particle density matrix. In this representation, the grand potential has a form that reminds us of density-functional theory,

$$
\begin{aligned}
\Omega_{\beta, \mu}(\hat{h}+\hat{W}) & =\min _{\left|\psi_{n}\right\rangle, f_{n} \in[0,1]} \operatorname{stat}\left\{\sum_{n} f_{n}\left\langle\psi_{n}|\hat{h}| \psi_{n}\right\rangle\right. \\
& +F_{\beta}^{\hat{W}}\left[\sum_{n}\left|\psi_{n}\right\rangle f_{n}\left\langle\psi_{n}\right|\right]-\mu \sum_{n} f_{n} \\
& \left.-\sum_{m, n} \Lambda_{m, n}\left(\left\langle\psi_{n} \mid \psi_{m}\right\rangle-\delta_{n, m}\right)\right\} .
\end{aligned}
$$


Note, that we used the symbol $\boldsymbol{\Lambda}$ here in a different context than before: Instead of constraining many-particle wave functions to be orthonormal, here the orthonormality is enforced for one-particle wave functions. The main difference of Eq. (11) from the corresponding expression for the density-functional theory is that densitymatrix functional theory uses the true kinetic energy, while density-functional theory uses the kinetic energy of a non-interacting electron gas.

\section{B. Kadanoff-Baym functional}

After having introduced the expression of the grand potential using the density-matrix functional, let us now turn to the alternative formulation in terms of Green's functions.

Luttinger and Ward ${ }^{12}$ have shown that the grand potential can also be expressed as a functional of the Matsubara Green's function and the self energy,

$$
\Omega_{\beta, \mu}(\hat{h}+\hat{W})=\operatorname{stat}_{G, \Sigma} \Psi_{\beta, \mu}^{K B}[\boldsymbol{G}, \boldsymbol{\Sigma}, \boldsymbol{h}, \hat{W}],
$$

where $\Psi_{\beta, \mu}^{K B}$ is called the Kadanoff-Baym functional. We adopt this naming following Chitra and Kotliar ${ }^{19}$. The Green's function and the self energy are given at fermionic Matsubara frequencies $\omega_{\nu}=(2 \nu+1) \pi /(\hbar \beta)$, specified by an integer $\nu$, and obtained by a Fourier transformation

$$
G\left(i \omega_{\nu}\right)=\frac{1}{2} \int_{-\hbar \beta}^{\hbar \beta} d \tau G(\tau) \mathrm{e}^{i \omega_{\nu} \tau}
$$

from of their imaginary-time partners. ${ }^{29}$ The imaginarytime Green's function, obeying the stationary condition, is given by

$$
G_{\alpha, \beta}(\tau)=-\frac{1}{\hbar} \operatorname{Tr}\left\{\mathrm{e}^{-\beta(\hat{h}+\hat{W}-\mu \hat{N}-\Omega)} \mathcal{T}_{\tau}\left\{\hat{c}_{\alpha}(\tau) \hat{c}_{\beta}^{\dagger}(0)\right\}\right\},
$$

where

$$
\begin{aligned}
& \hat{c}_{\alpha}(\tau)=\mathrm{e}^{(\hat{h}+\hat{W}-\mu \hat{N}) \tau / \hbar} \hat{c}_{\alpha} \mathrm{e}^{-(\hat{h}+\hat{W}-\mu \hat{N}) \tau / \hbar} \\
& \hat{c}_{\alpha}^{\dagger}(\tau)=\mathrm{e}^{(\hat{h}+\hat{W}-\mu \hat{N}) \tau / \hbar} \hat{c}_{\alpha}^{\dagger} \mathrm{e}^{-(\hat{h}+\hat{W}-\mu \hat{N}) \tau / \hbar} .
\end{aligned}
$$

The self energy $\boldsymbol{\Sigma}(\tau)$ is defined ${ }^{29}$ such that it connects the two-particle Green's function to the one-particle Green's function in the equation of motion for the Green's functions by

$$
\begin{aligned}
& \sum_{\gamma} \int_{0}^{\hbar \beta} d \tau^{\prime} \Sigma_{\alpha, \gamma}\left(\tau-\tau^{\prime}\right) G_{\gamma, \beta}\left(\tau^{\prime}, 0\right) \\
= & \sum_{b, c, d} W_{\alpha, b, c, d}\left(\frac{-1}{\hbar}\right) \operatorname{Tr}\left\{\mathrm{e}^{-\beta(\hat{h}+\hat{W}-\mu \hat{N}-\Omega)}\right. \\
\times & \left.\mathcal{T}_{\tau}\left\{c_{b}^{\dagger}(\tau) \hat{c}_{c}(\tau) \hat{c}_{d}(\tau) \hat{c}_{\beta}^{\dagger}(0)\right\}\right\} .
\end{aligned}
$$

The Kadanoff-Baym functional ${ }^{12}$

$$
\begin{aligned}
& \Psi_{\beta, \mu}^{K B}[\boldsymbol{G}, \boldsymbol{\Sigma}, \boldsymbol{h}, \hat{W}]=\Phi_{\beta}^{L W}[\boldsymbol{G}, \hat{W}] \\
& \quad-\frac{1}{\beta} \sum_{\nu} \operatorname{Tr}\left\{\ln \left(1-\frac{1}{\left(i \hbar \omega_{\nu}+\mu\right) \mathbf{1}-\boldsymbol{h}} \boldsymbol{\Sigma}\left(i \omega_{\nu}\right)\right)\right. \\
& \left.+\boldsymbol{\Sigma}\left(i \omega_{\nu}\right) \boldsymbol{G}\left(i \omega_{\nu}\right)\right\}-\frac{1}{\beta} \operatorname{Tr}\left\{\ln \left(\mathbf{1}+\mathrm{e}^{-\beta(\boldsymbol{h}-\mathbf{1} \mu)}\right)\right\}
\end{aligned}
$$

is built such that Green's function Eq. (14) and self energy Eq. (17) result from the stationary conditions specified in Eq. (12).

The first term on the right-hand side in Eq. (18) is the Luttinger-Ward functional $\Phi_{\beta}^{L W}$. The Luttinger-Ward functional is a sum of all closed, connected and irreducible skeleton diagrams with the non-interacting propagator replaced by the fully interacting one. ${ }^{12,30}$ As is obvious from its diagrammatic definition, it is a functional of the Green's function and the interaction only but does not depend on the one-particle Hamiltonian which contains the external potential. In this respect it is "universal." Note that $\Phi_{\beta}^{L W}$ vanishes for a non-interacting system, that is for $\hat{W}=0$. Furthermore, the diagrammatic construction implies

$$
\frac{\beta \delta \Phi_{\beta}^{L W}[\boldsymbol{G}, \hat{W}]}{\delta G_{b, a}\left(i \omega_{\nu}\right)}=\Sigma_{a, b}\left(i \omega_{\nu}\right) .
$$

This equation is also equivalent with the stationary condition of the Kadanoff-Baym functional with respect to the Green's function. The stationary condition with respect to the self energy is

$$
\boldsymbol{G}\left(i \omega_{\nu}\right)=\left(\left(i \hbar \omega_{\nu}+\mu\right) \mathbf{1}-\boldsymbol{h}-\boldsymbol{\Sigma}\left(i \omega_{\nu}\right)\right)^{-1}
$$

This is just Dyson's equation.

\section{CONNECTING DENSITY-MATRIX FUNCTIONAL AND LUTTINGER-WARD FUNCTIONAL}

Before we connect the Luttinger-Ward functional to the density-matrix functional, we investigate the transformation properties of the Kadanoff-Baym functional under changes $\boldsymbol{\Delta}$ of the non-interacting Hamiltonian.

In Appendix B, we show that

$$
\begin{array}{r}
\Psi^{K B}[\boldsymbol{G}, \boldsymbol{\Sigma}, \boldsymbol{h}, \hat{W}]=\Psi^{K B}[\boldsymbol{G}, \boldsymbol{\Sigma}+\boldsymbol{\Delta}, \boldsymbol{h}-\boldsymbol{\Delta}, \hat{W}] \\
+\frac{1}{\beta} \sum_{\nu} \mathrm{e}^{i \beta \hbar \omega_{\nu} 0^{+}} \operatorname{Tr}\left\{\boldsymbol{G}\left(i \omega_{\nu}\right) \boldsymbol{\Delta}\right\} .
\end{array}
$$

The important feature of Eq. (21) is that it holds pointper-point and not only when the stationary conditions are satisfied. This allows one to choose the transformation depending on the actual value of the Green's function $\boldsymbol{G}$.

In the following, we will employ this invariance, i.e. Eq. (21), to connect the Kadanoff-Baym functional to 
the density-matrix functional by introducing a new noninteracting Hamiltonian, which eventually will be a functional of the density matrix.

A new non-interacting Hamiltonian $\overline{\boldsymbol{h}}[\boldsymbol{\rho}[\boldsymbol{G}]]=\boldsymbol{h}-$ $\boldsymbol{\Delta}[\boldsymbol{\rho}[\boldsymbol{G}]]$ as functional of the Green's function is obtained as follows: From the Green's function $\boldsymbol{G}$ we obtain the one-particle reduced density matrix via

$$
\boldsymbol{\rho}[\boldsymbol{G}]=\frac{1}{\beta} \sum_{\nu} \mathrm{e}^{i \beta \hbar \omega_{\nu} 0^{+}} \boldsymbol{G}\left(i \omega_{\nu}\right) .
$$

The (non-interacting) Hamiltonian $\hat{\bar{h}}$ is defined by requiring that it lead to the density matrix given by Eq. (22), i.e.:

$$
\boldsymbol{\rho}=\left[\mathbf{1}+\mathrm{e}^{\beta(\overline{\boldsymbol{h}}-\mu \mathbf{1})}\right]^{-1},
$$

Explicitly, it is given by

$$
\overline{\boldsymbol{h}}[\boldsymbol{\rho}]=\mu \mathbf{1}+\frac{1}{\beta} \ln \left[\frac{1-\boldsymbol{\rho}}{\rho}\right] .
$$

The identity is easily seen in the representation of natural orbitals, where all matrices are diagonal: Then, Eq. (23) is the expression for the Fermi distribution. The Hamiltonian $\overline{\boldsymbol{h}}$ is, in general, a non-local Hamiltonian.
The construction just described is practical only at finite temperatures. At zero temperature the spectrum of $\overline{\boldsymbol{h}}$ collapses to a single energy, namely the Fermi level.

The required change for the one-particle Hamiltonian is

$$
\Delta[\boldsymbol{\rho}]=\boldsymbol{h}-\overline{\boldsymbol{h}}=(\boldsymbol{h}-\mu \mathbf{1})-\frac{1}{\beta} \ln \left[\frac{\mathbf{1}-\boldsymbol{\rho}}{\boldsymbol{\rho}}\right]
$$

The transformation turns the grand potential of noninteracting electrons (last term in Eq. (18)) into a form that can be expressed conveniently by the natural orbitals $\left|\psi_{n}\right\rangle$ and their occupations $f_{n}$, i.e. the eigenvectors and eigenvalues of the reduced density matrix $\boldsymbol{\rho}$.

$$
\begin{aligned}
-\frac{1}{\beta} & \operatorname{Tr}\left\{\ln \left(1+\mathrm{e}^{-\beta(\overline{\boldsymbol{h}}-\mathbf{1} \mu)}\right)\right\} \\
& =\sum_{n} f_{n}\left\langle\psi_{n}|\hat{\bar{h}}| \psi_{n}\right\rangle-\mu \sum_{n} f_{n} \\
& +\frac{1}{\beta} \sum_{n}\left[f_{n} \ln \left(f_{n}\right)+\left(1-f_{n}\right) \ln \left(1-f_{n}\right)\right],
\end{aligned}
$$

With this, the Kadanoff-Baym functional can be rewritten as

$$
\begin{aligned}
\Psi_{\beta, \mu}^{K B}[\boldsymbol{G}, \boldsymbol{\Sigma}, \boldsymbol{h}, \hat{W}] & =\sum_{n} f_{n}\left\langle\psi_{n}|\hat{h}| \psi_{n}\right\rangle+\frac{1}{\beta} \sum_{n}\left(f_{n} \ln \left(f_{n}\right)+\left(1-f_{n}\right) \ln \left(1-f_{n}\right)\right)-\mu \sum_{n} f_{n} \\
& +\Phi_{\beta}^{L W}[\boldsymbol{G}, \hat{W}]-\frac{1}{\beta} \sum_{\nu} \mathrm{e}^{i \beta \hbar \omega_{\nu} 0^{+}} \operatorname{Tr}\left\{\ln \left[\mathbf{1}-\left(\left(i \hbar \omega_{\nu}+\mu\right) \mathbf{1}-\overline{\boldsymbol{h}}\right)^{-1}\left(\boldsymbol{h}+\boldsymbol{\Sigma}\left(i \omega_{\nu}\right)-\overline{\boldsymbol{h}}\right)\right]\right. \\
& \left.+\left(\boldsymbol{h}+\boldsymbol{\Sigma}\left(i \omega_{\nu}\right)-\overline{\boldsymbol{h}}\right) \boldsymbol{G}\left(i \omega_{\nu}\right)+\boldsymbol{G}\left(i \omega_{\nu}\right)(\overline{\boldsymbol{h}}-\boldsymbol{h})\right\}+\sum_{n} f_{n}\left\langle\psi_{n}|(\hat{\bar{h}}-\hat{h})| \psi_{n}\right\rangle
\end{aligned}
$$

where $\overline{\boldsymbol{h}}$, the natural orbitals $\left|\psi_{n}\right\rangle$ and the occupations $f_{n}$ are, via Eq. (22), functionals of the Green's function $\boldsymbol{G}$. The last term in Eq. (27) ensures that the first term is the expectation value of the true one-particle Hamiltonian and not the expectation value of $\hat{\bar{h}}$. The operator $\hat{\bar{h}}$ is defined via the matrix $\overline{\boldsymbol{h}}$ analogously to Eq. (2).

Note that the last term $\boldsymbol{G}\left(i \omega_{\nu}\right)(\overline{\boldsymbol{h}}-\boldsymbol{h})$ in the Matsubara sum in Eq. (27) can in principle be canceled against a similar contribution in the preceding term. It however also combines with the last term of Eq. (27) to a contribution that vanishes when the Green's function fulfills the density-matrix constraint. By keeping it explicitly we maintain the integrity of conceptually related entities and make the derivation more transparent.

For practical purposes, it will be convenient to rewrite the last two terms in Eq. (27) using the identity

$$
\begin{aligned}
- & \operatorname{Tr}\left\{\left(\boldsymbol{\rho}-\frac{1}{\beta} \sum_{\nu} \mathrm{e}^{i \beta \hbar \omega_{\nu} 0^{+}} \boldsymbol{G}\left(i \omega_{\nu}\right)\right)(\boldsymbol{h}-\overline{\boldsymbol{h}})\right\} \\
= & \frac{1}{\beta} \sum_{\nu} \operatorname{Tr}\left\{\left(\boldsymbol{G}\left(i \omega_{\nu}\right)-\frac{1}{\left(i \hbar \omega_{\nu}+\mu\right) \mathbf{1}-\overline{\boldsymbol{h}}}\right)\right. \\
& \times(\boldsymbol{h}-\overline{\boldsymbol{h}})\},
\end{aligned}
$$

because the latter has a converging Matsubara sum.

In the spirit of density-functional theory, we can treat the density matrix, that is the natural orbitals and the occupations, as independent variables that are, however, linked to the Green's function by a constraint that enforces Eq. (22).

This argument can also be turned around: Instead of constraining the density matrix to the Green's function during the optimization of the latter, we may as well perform a search over the density matrices. For each density matrix, the optimum Green's function is determined un- 
der a density-matrix constraint. This approach naturally leads to a density-matrix functional expressed as a constrained search over Green's functions and self-energies. The search over Green's functions is guided by a station- arity principle rather than by an extremum principle. form

$$
\Omega_{\beta, \mu}^{K B}[\hat{h}+\hat{W}]=\min _{\left|\psi_{n}\right\rangle, f_{n} \in[0,1]} \operatorname{stat}\left\{\sum_{n} f_{n}\left\langle\psi_{n}|\hat{h}| \psi_{n}\right\rangle+\tilde{F}_{\beta}^{\hat{W}}\left[\sum_{n}\left|\psi_{n}\right\rangle f_{n}\left\langle\psi_{n}\right|\right]-\mu \sum_{n} f_{n}-\sum_{m, n} \Lambda_{m, n}\left(\left\langle\psi_{n} \mid \psi_{m}\right\rangle-\delta_{m, n}\right)\right\}
$$

with

$$
\begin{aligned}
\tilde{F}_{\beta}^{W}[\boldsymbol{\rho}]= & \frac{1}{\beta} \operatorname{Tr}[\boldsymbol{\rho} \ln (\boldsymbol{\rho})+(\mathbf{1}-\boldsymbol{\rho}) \ln (\mathbf{1}-\boldsymbol{\rho})] \\
+ & \underset{\boldsymbol{h}^{\prime}}{\operatorname{stat} \operatorname{stat}_{\boldsymbol{G}}}\left\{\Phi_{\beta}^{L W}[\boldsymbol{G}, \hat{W}]-\frac{1}{\beta} \sum_{\nu} \operatorname{Tr}\left\{\ln \left[\mathbf{1}-\left(\left(i \hbar \omega_{\nu}+\mu\right) \mathbf{1}-\overline{\boldsymbol{h}}\right)^{-1}\left(\boldsymbol{h}^{\prime}+\boldsymbol{\Sigma}\left(i \omega_{\nu}\right)-\overline{\boldsymbol{h}}\right)\right]\right.\right. \\
& \left.\left.+\left(\boldsymbol{h}^{\prime}+\boldsymbol{\Sigma}\left(i \omega_{\nu}\right)-\overline{\boldsymbol{h}}\right) \boldsymbol{G}\left(i \omega_{\nu}\right)-\left[\boldsymbol{G}\left(i \omega_{\nu}\right)-\left(\left(i \hbar \omega_{\nu}+\mu\right) \mathbf{1}-\overline{\boldsymbol{h}}\right)^{-1}\right]\left(\boldsymbol{h}^{\prime}-\overline{\boldsymbol{h}}\right)\right\}\right\}
\end{aligned}
$$

where $\overline{\boldsymbol{h}}$ is a functional of the one-particle reduced density matrix $\rho$.

Let us explore the various terms in Eq. (30): The last term in the Matsubara sum of Eq. (30), $\frac{1}{\beta} \sum_{\nu}(\boldsymbol{G}-$ $\overline{\boldsymbol{G}})\left(\boldsymbol{h}^{\prime}-\overline{\boldsymbol{h}}\right)$ vanishes, when the Green's function $\boldsymbol{G}$ obeys the density-matrix constraint. $\bar{G}$ is a short hand for

$$
\overline{\boldsymbol{G}}\left(i \omega_{\nu}\right)=\left(\left(i \hbar \omega_{\nu}+\mu\right) \mathbf{1}-\overline{\boldsymbol{h}}\right)^{-1}
$$

The Luttinger-Ward functional combined with the remainder of the Matsubara sum corresponds the interaction part of the Kadanoff-Baym functional for an interacting system with a one-particle Hamiltonian $\overline{\boldsymbol{h}}$ instead of $\boldsymbol{h}$ and a self energy $\boldsymbol{h}^{\prime}+\boldsymbol{\Sigma}-\overline{\boldsymbol{h}}$.

In the derivation of Eq. (30), we exploited that the derivative of the functional inside the expression of Eq. (30) with respect to the one-particle Hamiltonian $\boldsymbol{h}^{\prime}$ (formerly $\boldsymbol{h}$ ) vanishes when the density-matrix constraint Eq. (22) is obeyed. Thus, the constraint that the Green's function is consistent with the density matrix can be imposed simply by requiring that the derivative with respect to $\boldsymbol{h}^{\prime}$ vanishes. We have added a prime to make it evident that $\boldsymbol{h}^{\prime}$ is a Lagrange multiplier.

Like the density-matrix functional $F_{\beta}^{\hat{W}}$ defined via a constrained search over many-particle wave functions, the functional $\tilde{F}_{\beta}^{\hat{W}}$ is universal, that is, it is independent of the one-particle Hamiltonian $\boldsymbol{h}$ of the physical system of interest and also independent of the chemical potential.

The fact that it is independent of the chemical potential is seen from the definition of $\overline{\boldsymbol{h}}$ and from the fact that $\boldsymbol{h}^{\prime}$ is a Lagrange multiplier, which can absorb a constant term. Thus, we may simply set the chemical potential in Eq. (30) and in Eq. (24) to zero.

With Eq. (30) and Eq. (8) we have two very different representations of the density-matrix functional. It is most important to realize, however, that both representations are exact representations of the same densitymatrix functional. This becomes obvious from the comparison of Eq. (29) with Eq. (11), from the fact that both representations are universal and from the fact that, up to now, all calculations have been free of any approximation. The representation Eq. (30) is novel and makes the link between Green's-function-based approaches and those based on many-particle wave functions.

Having derived a density-matrix functional from Green's functions and self-energies, we have introduced a true minimum principle into the Green's function world. The search for the physical one-particle density matrix has a true minimum principle. The determination of the minimum is substantially simpler than the search for a saddle point.

Some caution is still required. The minimum property follows from the representation Eq. (8) for the densitymatrix functional but is obviously guaranteed for the exact functional only, rather than for every conceivable approximation. We consider this as a mathematical caveat, that will not be relevant in practice: Approximations that change the total energy surface qualitatively can probably not be considered as reliable.

One should also note that it is unavoidable that one part of the optimization still requires a saddle point search. This part has been moved into the evaluation of the density-matrix functional from Green's functions and self energies.

For the sake of completeness, let us note here the sta- 
tionary conditions of Eq. (30)

$$
\begin{aligned}
\boldsymbol{\Sigma}\left(i \omega_{\nu}\right) & =\beta \frac{\delta \Phi_{\beta}^{L W}[\boldsymbol{G}, \hat{W}]}{\delta \boldsymbol{G}\left(i \omega_{\nu}\right)} \\
\boldsymbol{\rho} & =\frac{1}{\beta} \sum_{\nu} \mathrm{e}^{i \beta \hbar \omega_{\nu} 0^{+}} \boldsymbol{G}\left(i \omega_{\nu}\right) \\
\boldsymbol{G}\left(i \omega_{\nu}\right) & =\left[\left(i \hbar \omega_{\nu}+\mu\right) \mathbf{1}-\boldsymbol{h}^{\prime}-\boldsymbol{\Sigma}\left(i \omega_{\nu}\right)\right]^{-1} .
\end{aligned}
$$

Furthermore, as shown in Appendix C, the functional derivative of the density-matrix functional Eq. (30) is

$$
\frac{\partial \tilde{F}_{\beta}^{\hat{W}}[\boldsymbol{\rho}]}{\partial \boldsymbol{\rho}}=-\left(\boldsymbol{h}^{\prime}-\mu\right) .
$$

Combining this with the stationary condition of the Kadanoff-Baym functional Eq. (29) with respect to variations of the density matrix we obtain

$$
\boldsymbol{h}=\boldsymbol{h}^{\prime}
$$

as condition for the minimum.

The expression in the density-matrix functional $\tilde{F}_{\beta}^{\hat{W}}$ is not an extremum with respect to the self energy or the Green's function. Therefore, we cannot use a gradientfollowing technique, but we have to resort to a selfconsistency scheme defined by the following steps.

1. First one constructs the Hamiltonian $\overline{\boldsymbol{h}}$ from the specified density matrix $\boldsymbol{\rho}$ via Eq. (24). The value for the chemical potential is arbitrary and can be set to zero.

2. The initial self energy is set to zero and the Lagrange multiplier $\boldsymbol{h}^{\prime}$ is set equal to $\overline{\boldsymbol{h}}$. This ensures that the initial Green's function

$$
\boldsymbol{G}\left(i \omega_{\nu}\right)=\left[\left(i \hbar \omega_{\nu}+\mu\right) \mathbf{1}-\boldsymbol{h}^{\prime}-\boldsymbol{\Sigma}\left(i \omega_{\nu}\right)\right]^{-1}
$$

is equal to $\overline{\boldsymbol{G}}$ defined in Eq. (31), which satisfies the density-matrix constraint.

3. Next we evaluate a new self-energy from Eq. (19).

4. The new self energy and $\boldsymbol{h}^{\prime}$ define a Green's function $\overline{\boldsymbol{G}}\left(i \omega_{\nu}\right)$ via Dyson's equation Eq. (37), which does not yet satisfy the density-matrix constraint. Now the Lagrange multiplier $\boldsymbol{h}^{\prime}$ is adjusted such that the constraint condition is fulfilled. This is done iteratively. In each iteration, the constraint equation is expanded to first order in the change $\delta \boldsymbol{h}^{\prime}$ of the Lagrange multiplier $\boldsymbol{h}^{\prime}$. This linearized constraint condition

$$
\frac{1}{\beta} \sum_{\nu} \mathrm{e}^{i \beta \hbar \omega 0^{+}}\left(\overline{\boldsymbol{G}}\left(i \omega_{\nu}\right)+\overline{\boldsymbol{G}}\left(i \omega_{\nu}\right) \delta \boldsymbol{h}^{\prime} \overline{\boldsymbol{G}}\left(i \omega_{\nu}\right)\right)=\boldsymbol{\rho}
$$

provides a correction $\delta \boldsymbol{h}^{\prime}$ to the Lagrange multiplier. The new Lagrange multiplier defines an improved Green's function $\overline{\boldsymbol{G}}\left(i \omega_{\nu}\right)$ via Eq. (37). This step 4 is repeated until the Lagrange multiplier is converged.

5. Once the constraint is obeyed, $\boldsymbol{G}$ is replaced by the converged $\overline{\boldsymbol{G}}$ and one returns to step 3 and recalculates the self energy.

6. When the loop of steps 3-5 is converged, the energy contribution from the density-matrix functional is evaluated from Eq. (30) and its derivative from Eq. (35).

\section{APPROXIMATIONS}

One of the major benefits of the new construction of the density-matrix functional defined in Eq. (30) is that it not only makes contact with density-functional theory but also with approximations defined diagrammatically in the context of Green's-function approaches.

\section{A. Relation to density-functional theory}

There is an intimate connection between densitymatrix functional theory and density-functional theory: It is easily shown that the density functional can be obtained from a constrained minimization of the densitymatrix functional. However, in order to embed an explicit treatment of many-particle correlations into the available density functional codes as in methods that link DFT with dynamical mean-field theory ${ }^{31-34}$, it is desirable to take the opposite route, namely to develop an approximate density-matrix functional that is consistent with a given density functional.

The grand potential in density-functional theory is ${ }^{11}$

$$
\begin{gathered}
\Omega_{\beta, \mu}\left[v_{\text {ext }}\right]=\min _{n}\left\{K_{s}[n]+\int d^{3} r v_{\text {ext }}(\vec{r}) n(\vec{r})\right. \\
\left.+E_{H}[n]+E_{x c, \beta}[n]-\mu \int d^{3} r n(\vec{r})\right\}
\end{gathered}
$$

where $K_{s}[n]$ is the intrinsic energy, i.e., kinetic energy and entropy term, of a non-interacting electron gas with density $n(\vec{r})$ at a temperature specified by $\beta$. The intrinsic energy of the interacting electrons contains in addition the Hartree energy $E_{H}[n]$ and the exchange correlation energy $E_{x c}[n]$. In Appendix D, we briefly review the adiabatic connection $^{35}$ to make the notation more explicit, with a particular emphasis on the extension to finite temperatures. The exchange-correlation energy $E_{x c, \beta}[n]$ contains at finite temperatures not only a contribution of the kinetic energy but also an additional correction to account for the entropy difference of the interacting and the non-interacting electron gas.

Comparing Eq. (39) with Eq. (7) of the density-matrix functional theory, we can construct a new density-matrix 
functional.

$$
\tilde{F}_{\beta}^{\hat{W}, D F T}[\boldsymbol{\rho}]=E_{H}[n]+E_{x c, \beta}[n]+K_{s}[n]-E_{k i n}[\boldsymbol{\rho}],
$$

where $n(\vec{r})$ is obtained from $\boldsymbol{\rho}$.

Even for the exact density functional, the functional $\tilde{F}_{\beta}^{\hat{W}, D F T}$ constructed in this way differs from the exact density-matrix functional $F_{\beta}^{\hat{W}}$ defined earlier in Eq. (8). The two functionals agree, however, for the physically relevant density matrices $\boldsymbol{\rho}[n]$, which are obtained from minimization of $F_{\beta}^{\hat{W}}[\boldsymbol{\rho}]$ for a fixed density $n(\vec{r})$, i.e.

$$
E_{k i n}[\boldsymbol{\rho}]+F_{\beta}^{\hat{W}, D F T}[\boldsymbol{\rho}]=\min _{\boldsymbol{\rho}^{\prime} \rightarrow n[\boldsymbol{\rho}]} E_{k i n}\left[\boldsymbol{\rho}^{\prime}\right]+F_{\beta}^{\hat{W}}\left[\boldsymbol{\rho}^{\prime}\right] .
$$

Thus, both functionals predict the same grand potentials.

Therefore, we consider this density-matrix functional derived from density-functional theory as a useful expression to obtain double-counting correction terms for the embedding of higher-level theories into density-functional theory with conventional, parametrized density functionals.

\section{B. Hartree-Fock Approximation}

The Hartree-Fock approach can be considered as resulting from the Ritz variational principle using a Slater determinant as a trial many-body wave function. Alternatively, the approach can be defined by the most simple truncation of the Luttinger-Ward functional. Keeping only first-order renormalized skeleton diagrams, we find

$$
\begin{array}{r}
\Phi^{L W, H F}[\boldsymbol{G}]=\frac{1}{2} \frac{1}{\beta^{2}} \sum_{\nu, \nu^{\prime}} e^{i \beta \hbar \omega_{\nu} 0^{+}} e^{i \beta \hbar \omega_{\nu^{\prime}} 0^{+}} \sum_{a, b, c, d} U_{a, b, d, c} \\
\times\left(G_{d, a}\left(i \omega_{\nu}\right) G_{c, b}\left(i \omega_{\nu^{\prime}}\right)-G_{c, a}\left(i \omega_{\nu}\right) G_{d, b}\left(i \omega_{\nu^{\prime}}\right)\right) .
\end{array}
$$

Carrying out the summations over the Matsubara frequencies, this trivially becomes a density-matrix functional.

After satisfying the stationary conditions Eqs. (32), (33) and (34), the density-matrix functional Eq. (30) in the Hartree-Fock approximation is

$$
\begin{aligned}
\tilde{F}_{\beta}^{\hat{W}, H F}[\boldsymbol{\rho}] & =\frac{1}{2} \sum_{a, b, c, d} U_{a, b, d, c}\left(\rho_{d, a} \rho_{c, b}-\rho_{c, a} \rho_{d, b}\right) \\
& +\frac{1}{\beta} \operatorname{Tr}[\boldsymbol{\rho} \ln (\boldsymbol{\rho})+(\mathbf{1}-\boldsymbol{\rho}) \ln (\mathbf{1}-\boldsymbol{\rho})],
\end{aligned}
$$

which consists of Hartree and exchange energy as well as an entropy contribution.

The Hartree-Fock self energy, obtained from Eq. (32) is frequency independent and equals $\Sigma_{a, b}^{H F}[\boldsymbol{\rho}]$, which has the form

$$
\Sigma_{a, b}^{H F}[\boldsymbol{\rho}]=\sum_{c, d}\left(U_{a, c, b, d}-U_{a, c, d, b}\right) \rho_{d, c} .
$$

The optimum Green's function, which satisfies Eq. (34), is

$$
\boldsymbol{G}^{H F}\left(i \omega_{\nu}\right)=\left[\left(i \hbar \omega_{\nu}+\mu\right) \mathbf{1}-\boldsymbol{h}^{\prime}-\boldsymbol{\Sigma}^{H F}[\boldsymbol{\rho}]\right]^{-1},
$$

where the Lagrange multiplier $\boldsymbol{h}^{\prime}$ is chosen such that the density-matrix constraint Eq. (33) is obeyed.

The Lagrange multiplier can also obtained from Eq. (35) by inserting the derivative of the density-matrix functional Eq. (43) with respect to the density matrix

$$
\boldsymbol{h}^{\prime}=\mu \mathbf{1}-\boldsymbol{\Sigma}^{H F}[\boldsymbol{\rho}]-k_{B} T \ln \frac{\boldsymbol{\rho}}{\mathbf{1}-\boldsymbol{\rho}} .
$$

This identifies the Hartree-Fock Green's function Eq. (45) with $\overline{\boldsymbol{G}}$ defined earlier in Eq. (31).

For a given one-particle Hamiltonian, the minimum condition Eq. (36) of the grand potential with respect to the density matrix provides us with the optimum density matrix

$$
\boldsymbol{\rho}=\left(\mathbf{1}+\mathrm{e}^{\beta\left(\boldsymbol{h}+\boldsymbol{\Sigma}^{H F}[\boldsymbol{\rho}]-\mu \mathbf{1}\right)}\right)^{-1},
$$

which must be solved self-consistently with Eq. (44).

The Hartree-Fock approximation is closely related to the $\mathrm{LDA}+U$ method $^{36,37}$ and the hybrid density functionals ${ }^{38,39}$, which replace part of the local exchange of DFT by the exact Fock term. Both methods can be considered as hybrid methods of density-functional theory and the Hartree-Fock approximation in the sense described earlier. ${ }^{40}$

The Hartree-Fock approximation is the most simple example of a so-called "conserving approximation" in the sense of Baym and Kadanoff. ${ }^{13,14}$ Conserving approximations result from truncations of the LuttingerWard functional and respect the macroscopic conservation laws resulting from continuous symmetries of the Hamiltonian. There have been a variety of attempts to systematically improve on the Hartree-Fock approximation by taking into account additional classes of diagrams from self-consistent second-order perturbation theory, the random-phase approximation and the self-consistent $G W$ approach, ${ }^{41}$ over the T-matrix or fluctuation-exchange approximation ${ }^{13,42}$ to rather complex theories such as the Parquet equations. ${ }^{42,43} \mathrm{In}$ the present context, they provide approximate, namely perturbatively defined, density-matrix functionals which should be valid for "weakly correlated" systems.

\section{Density-matrix functionals without Green's function optimization}

One of the main computational obstacles related to density functionals derived from the Kadanoff-Baym functional is that they are defined through a set of selfconsistency equations involving Green's functions and self energies. An algorithmic expression for the densitymatrix functional that can be evaluated directly, as that 
of the Hartree-Fock calculation, would thus be highly desirable.

Here, we present such an approximation, which results from the neglect of dynamical effects to second and higher orders (NDE2). To obtain this approximation, we rewrite the density-matrix functional Eq. (30) as

$$
\begin{aligned}
\tilde{F}_{\beta}^{\hat{W}}[\boldsymbol{\rho}] & =\frac{1}{\beta} \operatorname{Tr}[\boldsymbol{\rho} \ln (\boldsymbol{\rho})+(\mathbf{1}-\boldsymbol{\rho}) \ln (\mathbf{1}-\boldsymbol{\rho})] \\
& +\Phi_{\beta}^{L W}[\overline{\boldsymbol{G}}[\boldsymbol{\rho}], \hat{W}]+\Delta \tilde{F}_{\beta}^{\hat{W}}[\boldsymbol{\rho}],
\end{aligned}
$$

where the argument $\overline{\boldsymbol{G}}[\boldsymbol{\rho}]$ of the Luttinger-Ward functional is a direct functional of the density matrix defined in Eq. (31). The remainder

$$
\begin{aligned}
\Delta \tilde{F}_{\beta}^{\hat{W}}[\boldsymbol{\rho}] & =\Phi_{\beta}^{L W}[\boldsymbol{G}, \hat{W}]-\Phi_{\beta}^{L W}[\overline{\boldsymbol{G}}, \hat{W}] \\
& +\sum_{\nu} \operatorname{Tr}\left\{\left.\frac{\partial \Phi_{\beta}^{L W}}{\partial \boldsymbol{G}\left(i \omega_{\nu}\right)}\right|_{\boldsymbol{G}}\left(\overline{\boldsymbol{G}}\left(i \omega_{\nu}\right)-\boldsymbol{G}\left(i \omega_{\nu}\right)\right)\right\} \\
& +\frac{1}{\beta} \sum_{\nu} \operatorname{Tr}\left\{\sum_{n=2}^{\infty} \frac{1}{n}\left(\mathbf{1}-\overline{\boldsymbol{G}}\left(i \omega_{\nu}\right) \boldsymbol{G}^{-1}\left(i \omega_{\nu}\right)\right)^{n}\right\},
\end{aligned}
$$

contains only terms that are quadratic or of higher order in $\boldsymbol{G}[\boldsymbol{\rho}]-\overline{\boldsymbol{G}}[\boldsymbol{\rho}]$, where $\boldsymbol{G}=\boldsymbol{G}[\boldsymbol{\rho}]$ is the Green's function that obeys the stationary conditions for the specified density matrix $\rho$.

If the dynamic correlations embodied in the deviation $\boldsymbol{G}[\boldsymbol{\rho}]-\overline{\boldsymbol{G}}[\boldsymbol{\rho}]$ are small, we can ignore the remain$\operatorname{der} \Delta \tilde{F}_{\beta}^{\hat{W}}[\boldsymbol{\rho}]$. This constitutes the NDE2 approximation, which has the advantage that $\bar{G}$ is obtained directly from the density matrix. Thus, the algorithmic complexity of NDE2 is similar to that of the Hartree-Fock approximation, in the sense that self-consistency loops are avoided and that only Green's functions with static self energies need to be considered. In contrast to the Hartree Fock approximation, however, it allows to systematically include higher-order terms in the interaction through the choice of the Luttinger-Ward functional. The NDE2 approximation is compatible with any approximation of the Luttinger-Ward functional.

The NDE2 approximation only affects the difference between two Green's function with the same density matrix. In contrast to expansions in the interaction, the terms, which are ignored in the NDE2 approximation do not affect the kinetic energy, the electron density nor the exchange hole. The approximations of NDE2 are from the outset limited strictly to the shape of the correlation hole. Thus, the NDE2 approximation may profit from a preservation of these sum rules.

Furthermore, the Green's function entering the Luttinger-Ward functional is a Green's function for a non local but static potential. This simplifies the calculations to some extent.

Thus, the apparent advantage of the NDE2 approximation is that it allows us to systematically construct approximations of the density matrix functional Eq. (8) from established many-body theories, keeping the complexity within a reasonable margin. To the best of our knowledge, the NDE2 is the first such approximation.

\section{Dynamical mean-field theory}

Dynamical mean-field theory can be defined as an approximation to the Luttinger-Ward functional and represents a non-perturbative conserving approximation. ${ }^{44}$

In dynamical mean-field theory, one usually limits the interaction to site-local terms. That is, clusters $\mathcal{C}_{R}$ of local orbitals are defined that we name correlated Hilbert spaces, and the interaction tensor $U_{a, b, c, d}$ is limited to these local Hilbert spaces. This amounts to the approximation

$$
\hat{W} \approx \sum_{R} \hat{W}_{R}
$$

with

$$
\hat{W}_{R}=\frac{1}{2} \sum_{a, b, c, d \in \mathcal{C}_{R}} U_{a, b, d, c} \hat{c}_{a}^{\dagger} \hat{c}_{b}^{\dagger} \hat{c}_{c} \hat{c}_{d} .
$$

The resulting Hamiltonian is that of a multi-band Hubbard model. In order to compensate for this truncation, the U-tensor elements are usually scaled down.

The Luttinger-Ward functional is only sensitive to the Green's-function matrix elements that are directly connected to the U-tensor elements. Thus, the self energy, which is given as the derivative of the Luttinger-Ward functional by Eq. (19), only acts on the union of the correlated Hilbert spaces.

The defining approximation of dynamical mean-field theory, ontop of the restriction to Hubbard-like Hamiltonians, is the local approximation of the self energy, which divides the self energy into a sum of local terms. Formulated in terms of the Luttinger-Ward functional, this means that the corresponding Luttinger-Ward functional is a sum of terms

$$
\Phi_{\beta}^{L W}\left[\boldsymbol{G}, \sum_{R} \hat{W}_{R}\right] \approx \sum_{R} \Phi_{\beta}^{L W}\left[\boldsymbol{G}, \hat{W}_{R}\right] .
$$

Because only Green's function elements contribute to the Luttinger-Ward functional that are connected to an interaction, this approximation implies that each term $\Phi_{\beta}^{L W}\left[\boldsymbol{G}, \hat{W}_{R}\right]$ is sensitive only to those elements of the Green's function that connect orbitals within the same local cluster $\mathcal{C}_{R}$.

In this approximation, the self energy

$$
\boldsymbol{\Sigma}\left(i \omega_{\nu}\right)=\sum_{R} \beta \frac{\delta \Phi_{\beta}^{L W}\left[\boldsymbol{G}, \hat{W}_{R}\right]}{\delta \boldsymbol{G}\left(i \omega_{\nu}\right)} .
$$

is a sum over the correlated Hilbert spaces.

While each term in the sum of Eq. (53) is formally a matrix in the full one-particle Hilbert space, the self energy can also be written as the direct sum

$$
\Sigma\left(i \omega_{\nu}\right)=\bigoplus_{R} \Sigma_{R}\left(i \omega_{\nu}\right)
$$


of local self energies

$$
\boldsymbol{\Sigma}_{R}\left(i \omega_{\nu}\right)=\beta \frac{\delta \Phi_{\beta}^{L W}\left[\boldsymbol{G}_{R}, \hat{W}_{R}\right]}{\delta \boldsymbol{G}_{R}\left(i \omega_{\nu}\right)} .
$$

where each local self energy $\boldsymbol{\Sigma}_{R}$ or Green's function $\boldsymbol{G}_{R}$ is a matrix with finite dimension in the specific correlated Hilbert space $\mathcal{C}_{R}$. On the correlated Hilbert space $\mathcal{C}_{R}$, the local Green's function $\boldsymbol{G}_{R}$ is identical to $\boldsymbol{G}$ and the local self energy $\boldsymbol{\Sigma}_{R}$ is identical to $\boldsymbol{\Sigma}$.

As a result of the local approximation of the self energy, the latter has no dependence on the reciprocal wave vector in a representation of Bloch waves.

When dynamical mean-field theory is formulated as limiting theory for infinite coordination number ${ }^{45}$, Müller-Hartmann has shown for a class of Hamiltonians with non-local electrostatic interaction that the Hartree energy remains finite, while the non-local exchangecorrelation energy vanishes ${ }^{46}$. Because the Hartree energy, like the exchange energy, is a direct functional of the density matrix, these terms are easily separated out in a density-matrix functional framework, so that they do not add to the complexity of the formalism.

\section{FORCES AND SPECTRAL PROPERTIES}

\section{A. Forces}

The force $F_{i}$ on the atomic coordinate $R_{i}$ is given by the derivative of the Helmholtz free energy $A_{\beta, N}:=$ $\Omega_{\beta, \mu}+\mu N$. The Helmholtz free energy is obtained from Eq. (29) as

$$
A_{\beta, N}(\vec{R})=\operatorname{Tr}[\boldsymbol{\rho}(\vec{R}) \boldsymbol{h}(\vec{R})]+\tilde{F}_{\beta}^{\hat{W}(\vec{R})}[\boldsymbol{\rho}(\vec{R})],
$$

where $\rho(\vec{R})$ fulfills the minimum and stationary conditions. The atomic positions enter via the Hamiltonian matrix elements, i.e. via $\boldsymbol{h}$ and $U_{a, b, c, d}$. The corresponding force is therefore

$$
\begin{aligned}
F_{i} & =-\operatorname{Tr}\left[\frac{\partial \boldsymbol{h}}{\partial R_{i}} \boldsymbol{\rho}\right]-\sum_{a, b, c, d} \frac{\partial \Phi_{\beta}^{L W}}{\partial U_{a, b, c, d}} \frac{\partial U_{a, b, c, d}}{\partial R_{i}} \\
& -\operatorname{Tr}\left[\left(\boldsymbol{h}+\frac{\partial \tilde{F}_{\beta}^{\hat{W}}}{\partial \boldsymbol{\rho}}\right) \frac{\partial \boldsymbol{\rho}}{\partial R_{i}}\right]
\end{aligned}
$$

The first two terms can be identified with a HellmannFeynman force, as they do not depend on the electronic degrees of freedom, namely the density matrix. Usually the Hellmann-Feynman force is identified with the electrostatic force on the nucleus, which is of limited practical value in electronic structure calculations. If the basisset depends directly on the atomic positions, however, this dependency enters into derivative of the Hamiltonian and the U-tensor. These terms are often called Pulay forces ${ }^{47}$. Interestingly, the corresponding contribution from the Utensor to the Pulay force only enters through the partial derivative of the Luttinger-Ward functional, which can be seen from Eq. (30).

The terms on the second line vanish for the optimum density matrix, because the prefactor is directly related to stationary conditions Eq. (36) and Eq. (35). Note, that the trace of the density matrix is the electron number, which does not depend on the atomic positions.

It has been found that the Hellmann-Feynman forces, even with the Pulay forces, are very sensitive to the quality of the convergence, i.e. on how well the stationary conditions are obeyed. This is due to the fact that, while the energy only depends in second order on a deviation from the stationary point, the forces are already sensitive to the first order. This dependence can be reduced by the terms on the second line. The most complex approach is to determine the linear response of the density matrix with respect to atomic positions. This route is not economical due to the effort for a linear response calculation.

However, already approximations for the derivative of the density matrix improve the convergence properties dramatically. This has led to the development of Andersen's force theorem ${ }^{48}$ for density-functional theory, where, for example, the electrons within a atom-centered sphere are displaced with the nucleus.

In the fictitious-Lagrangian approach to $a b$ initio molecular dynamics ${ }^{27}$, a completely different route is taken. Here, electronic degrees of freedom and the atomic positions are treated on the same footing, and both are propagated according to Newton's equations of motion. This implies that the forces used in this approach are partial derivatives and not total derivatives with respect to atomic positions. This implies that the contribution of the electronic response to the force Eq. (57) must be excluded. In a certain sense, the electron dynamics takes care of the linear response of the density matrix. Thus, only the Hellmann-Feynman and Pulay terms need to be evaluated. The fictitious Lagrangian approach, however, rests on the existence of a true minium principle, and establishing such a minimum principle has been one of the major motivations for this work.

\section{B. Accessing the single-particle excitation spectrum}

As outlined at the end of Sec. (III), the density-matrix functional Eq. (30) together with the self-consistency equations (32)-(34) describes an algorithm to calculate a self energy respectively the Green's function corresponding to a given density matrix.

Let us discuss this feature in some more detail: The first observation is that the algorithm can be applied to any $N$-representable density matrix. This allows one to employ one approximation for the density-matrix functional used to optimize the density matrix, and another one to obtain the single-particle dynamics from the resulting density matrix. Thus, one can exploit that some 
approximations are particularly suited for the total energies, while others have their strengths in the spectral properties.

For the optimization of the density matrix and atomic positions, one may apply an algorithm that never resorts to Green's functions and self energies. Here, one can adopt efficient algorithms that exploit the minimum principle. The link to the wave-function based formulation of density-matrix functional theory allows one to use functionals resting on Eq. (8), such as the one described earlier ${ }^{40}$ or the parametrized density matrix functionals $^{7-10}$ that refer neither to the many-particle wave function nor the Green's function. Another promising approach is the NDE2 approximation described in Sec. IV C, because it avoids self-consistency of Green's functions, while allowing us to make close contact to the method used to extract spectral properties.

Once a density matrix has been obtained, one adopts, in a final step, a favorite approximation to the LuttingerWard functional to extract the Green's function corresponding to that density matrix. This is done following the procedure outlined at the end of Sec. III. One such approximation to obtain spectral properties is dynamical mean-field theory, which is obtained by invoking the local approximation described in Sec. IV D.

It is important to emphasize here that the approximation used to obtain the density matrix and the approximation for the Luttinger-Ward functional used for the calculation of spectral properties need not be related in any way. For example, one can use densityfunctional theory to calculate a density matrix, and a Hartree-Fock functional for the Green's function, thereby producing genuine quasi-particle bandstructures without resorting to Koopman's theorem. ${ }^{49,50}$ Alternatively, one can employ the Luttinger-Ward functional of the dynamical mean-field theory at this point, which produces an approximation that at first sight seems to be the well-established density-functional-theory + dynamicalmean-field theory (DFT+DMFT) approach.

There are, however, important differences between our approach and the conventional setup of DFT+DMFT. In the DFT+DMFT hybrid approaches, the non-interacting Hamiltonian is extracted from a non-interacting electron gas with the same electron density as the interacting electron gas. While the Kohn-Sham bands provide a surprisingly good description of quasi-particle bandstructures, there is no apparent conceptual connection with the noninteracting part of the Hamiltonian entering the dynamical mean-field theory. Density-matrix functional theory, on the contrary, is at an advantage, because it uses a one-particle Hamiltonian $\boldsymbol{h}^{\prime}$ that is linked to the interacting electrons: It is the Lagrange multiplier obtained via Eq. (34) and Eq. (33) from the density matrix constraint for the density matrix of the interacting electron gas. As a result, the spectra obtained in the final step from the rDMFT method can be interpreted directly as physical excitation spectra, without the well-known interpretation problems of Kohn-Sham spectra.
If such a hybrid scheme is built into a DFT environment, there is a fairly well defined expression for the double counting term within the DFT+rDMFT approach. ${ }^{40}$ Furthermore, the double-counting term, only enters the first step, namely the determination of the density matrix. For the final step of calculating the Green's function from a given density matrix, double-counting corrections are not even required.

\section{CONCLUSIONS}

A link between Green's-function diagrammatic techniques and the reduced density-matrix functional theory has been established by a re-formulation of the densitymatrix functional involving a constrained search over single-particle Green's functions. This constrained search is equivalent to solving the corresponding stationarity conditions and can be performed in practice by setting up a self-consistency scheme. Diagrammatic weak-coupling approaches as well as the non-perturbative dynamical mean-field theory are obtained as specific approximations of the density-matrix functional in this way.

The reformulation of diagrammatic approximations within the context of density-matrix functional theory is of great importance as the latter provides a true minimum principle. This is opposed to "dynamical" functionals, i.e. functionals of the Green's-function or the self-energy which are generally not convex. Attempts to enforce the convexity by modifying the Kadanoff-Baym functional or other dynamical functionals would require modifying their global free-energy landscape in a qualitative manner and will thus most likely also distort the physically relevant properties of the functionals.

As the one-particle density matrix is obtained by a Matsubara-frequency summation of the single-particle Green's function, the density-matrix functional theory can be seen as an approach where a certain hypersurface in the space of electronic degrees of freedom is selected. This hypersurface picks those "static" degrees of freedom that result in a convex free-energy landscape while the free energy, or the grand potential, shows the "wrong" curvature along "dynamical" coordinates orthogonal to this hypersurface.

Clearly, convexity can be shown rigorously for the exact density-matrix functional only and is not necessarily true for approximations. Note that this is the same situation as in DFT: Many rigorous properties of exact density-functional theory do not carry over to approximate density functionals, e.g. to the LDA functional.

It is obvious, however, that a convex free-energy landscape is obtained much more easily in an approximation to an exact functional that is convex already. That is, one would expect that reliable approximations inherit the minimum property from the exact rDMFT theory. Or stated differently, one would reject diagrammatic approximations which do qualitatively change the topology of the rDMFT free-energy surface or even the local free- 
energy surface close to the physical point.

The case of dynamical mean-field theory is particularly interesting. Numerical work related to the question of convexity of the density-matrix functional derived from dynamical mean-field theory is in progress. Another exciting line of further development is to completely avoid the computation of the spectral function within dynamical mean-field theory, i.e., to set up a self-consistent rDMFT scheme involving static ground-state or thermal properties only, such as energies, reduced density matrices or forces. Here, one would profit from a formulation based on a minimum principle during the search for selfconsistency while spectral properties are still accessible in a final post-processing step as outlined in Sec. VB.

The price one has to pay is that one now needs to adjust the full one-particle Hamiltonian $\boldsymbol{h}^{\prime}$ together with the single-particle self energy to fulfill the density-matrix constraint. The complexity of this step is, however, not higher than that of a dynamical-mean-field-theory selfconsistency without charge optimization.

\section{ACKNOWLEDGMENTS}

Financial support by the Deutsche Forschungsgemeinschaft through FOR 1346 is gratefully acknowledged.

\section{Appendix A: Convexity of the density-matrix functional}

Here we show that the density-matrix functional defined by Eq. (8) as

$$
\begin{aligned}
F[\boldsymbol{\rho}]= & \min _{P_{j} \geq 0,\left|\Phi_{j}\right\rangle \tilde{\boldsymbol{h}}, \boldsymbol{\Lambda}, \lambda} \operatorname{stat}\left\{\sum_{j} P_{j}\left\langle\Phi_{j}|\hat{W}| \Phi_{j}\right\rangle+\frac{1}{\beta} \sum_{j} P_{j} \ln \left[P_{j}\right]\right. \\
& \left.-\sum_{i, j} \Lambda_{i, j}\left(\left\langle\Phi_{j} \mid \Phi_{i}\right\rangle-\delta_{j, i}\right)-\lambda\left(\sum_{j} P_{j}-1\right)+\sum_{a, b} \tilde{h}_{a, b}\left(\sum_{j} P_{j}\left\langle\Phi_{j}\left|\hat{c}_{a}^{\dagger} \hat{c}_{b}\right| \Phi_{j}\right\rangle-\rho_{b, a}\right)\right\}
\end{aligned}
$$

is convex. That is

$$
F\left[(1-\lambda) \boldsymbol{\rho}^{A}+\lambda \boldsymbol{\rho}^{B}\right] \leq(1-\lambda) F\left[\boldsymbol{\rho}^{A}\right]+\lambda F\left[\boldsymbol{\rho}^{B}\right]
$$

The proof is facilitated by the fact that the one-particle density matrix and all expectation values depend only linearly on the many-particle density matrix.

Before we start, let us introduce a few quantities: The constrained search for the reduced one-particle density matrix $\boldsymbol{\rho}^{A}$ leads to an optimum ensemble $\left\{\left|\Phi_{j}^{A}\right\rangle, P_{j}^{A}\right\}$ characterized by the many-particle density matrix $\hat{\Gamma}^{A}=$ $\sum_{j}\left|\Phi_{j}^{A}\right\rangle P_{j}^{A}\left\langle\Phi_{j}^{A}\right|$. Analogously, the many-particle density matrix $\hat{\Gamma}^{B}$ for the ensemble $\left\{\left|\Phi_{j}^{B}\right\rangle, P_{j}^{B}\right\}$ is connected to the one-particle reduced density matrix $\boldsymbol{\rho}^{B}$. We define the $\lambda$-dependent many-particle density matrix

$$
\hat{\Gamma}(\lambda):=(1-\lambda) \hat{\Gamma}^{A}+\lambda \hat{\Gamma}^{B} .
$$

which linearly connects the two ensembles. Diagonalization of $\hat{\Gamma}(\lambda)$ yields the many-particle wave functions $\left|\Phi_{j}(\lambda)\right\rangle$ and the eigenvalues $P_{j}(\lambda)$.

At first, we show that the many-particle density matrix $\hat{\Gamma}(\lambda)$ obeys the constraints required for the optimization. These constraints are (1) the orthonormality of the manyparticle wave functions, (2) the normalization constraint of the probabilities, (3) the requirements that the all probabilities are positive, and (4) the density-matrix constraint. Finally, (5), the ensemble wave functions must be antisymmetric under particle exchange.

The orthonormality of the many-particle wave functions $\left|\Phi_{j}(\lambda)\right\rangle$ in the ensemble follows directly from the fact that they are eigenstates of a Hermitian operator $\hat{\Gamma}(\lambda)$. To be precise, the eigenstates can be chosen to be orthonormal.

The normalization of the probabilities, i.e., $\sum_{j} P_{j}=1$, can be expressed as $\operatorname{Tr}[\hat{\Gamma}(\lambda)]=1$. It is easily shown that

$$
\begin{aligned}
\operatorname{Tr}[\hat{\Gamma}(\lambda)] & =\operatorname{Tr}\left[(1-\lambda) \hat{\Gamma}^{A}+\lambda \hat{\Gamma}^{B}\right] \\
& =(1-\lambda) \operatorname{Tr}\left[\hat{\Gamma}^{A}\right]+\lambda \operatorname{Tr}\left[\hat{\Gamma}^{B}\right]=1,
\end{aligned}
$$

which proves that $\hat{\Gamma}(\lambda)$ obeys the normalization constraint.

The positive definiteness of $\hat{\Gamma}(\lambda)$, i.e. $P_{j}(\lambda) \geq 0$, follows directly from the positive definiteness of $\hat{\Gamma}^{A}$ and $\hat{\Gamma}^{B}$. We need to show that for any many-particle state $|\Psi\rangle$ the expectation value $\langle\Psi|\hat{\Gamma}(\lambda)| \Psi\rangle$ is non-negative. We obtain

$$
\langle\Psi|\hat{\Gamma}(\lambda)| \Psi\rangle=(1-\lambda)\left\langle\Psi\left|\hat{\Gamma}^{A}\right| \Psi\right\rangle+\lambda\left\langle\Psi\left|\hat{\Gamma}^{B}\right| \Psi\right\rangle \geq 0,
$$

if $\left\langle\Psi\left|\hat{\Gamma}^{A}\right| \Psi\right\rangle \geq 0$ and $\left\langle\Psi\left|\hat{\Gamma}^{B}\right| \Psi\right\rangle \geq 0$, and if $0 \leq \lambda \leq 1$.

Next, we need to show that the density-matrix constraint

$$
\rho_{\beta, \alpha}(\lambda)=\operatorname{Tr}\left[\hat{\Gamma}(\lambda) \hat{c}_{\alpha}^{\dagger} \hat{c}_{\beta}\right]
$$

is obeyed. It is verified as follows

$$
\begin{aligned}
\operatorname{Tr}\left[\hat{\Gamma}(\lambda) \hat{c}_{\alpha}^{\dagger} \hat{c}_{\beta}\right] & =\operatorname{Tr}\left[\left((1-\lambda) \hat{\Gamma}^{A}+\lambda \hat{\Gamma}^{B}\right) \hat{c}_{\alpha}^{\dagger} \hat{c}_{\beta}\right] \\
& =(1-\lambda) \operatorname{Tr}\left[\hat{\Gamma}^{A} \hat{c}_{\alpha}^{\dagger} \hat{c}_{\beta}\right]+\lambda \operatorname{Tr}\left[\hat{\Gamma}^{B} \hat{c}_{\alpha}^{\dagger} \hat{c}_{\beta}\right] \\
& =(1-\lambda) \rho_{\beta, \alpha}^{A}+\lambda \rho_{\beta, \alpha}^{B}=\rho_{\beta, \alpha}(\lambda) .
\end{aligned}
$$


Finally, the antisymmetry also carries over from the end-points due to the linearity of Eq. (A3).

As all the constraints are obeyed by $\hat{\Gamma}(\lambda)$, we obtain an upper bound for the density-matrix functional by evaluating the energy contributions of $(\mathrm{A})$ with $\hat{\Gamma}(\lambda)$, i.e.

$$
F[\boldsymbol{\rho}(\lambda)] \leq \operatorname{Tr}[\hat{\Gamma}(\lambda) \hat{W}]+\frac{1}{\beta} \operatorname{Tr}[\hat{\Gamma}(\lambda) \ln (\hat{\Gamma}(\lambda))]
$$

The interaction energy depends linearly on the manyparticle density matrix, so that

$$
\operatorname{Tr}[\hat{\Gamma}(\lambda) \hat{W}]=(1-\lambda) \operatorname{Tr}\left[\hat{\Gamma}^{A} \hat{W}\right]+\lambda \operatorname{Tr}\left[\hat{\Gamma}^{B} \hat{W}\right],
$$

and the entropy $-k_{B} \operatorname{Tr}[\hat{\Gamma} \ln (\hat{\Gamma})]$ is concave ${ }^{51}$.

With

$$
\begin{gathered}
F\left[\boldsymbol{\rho}^{A}\right]=\operatorname{Tr}\left[\hat{\Gamma}^{A} \hat{W}\right]+\frac{1}{\beta} \operatorname{Tr}\left[\hat{\Gamma}^{A} \ln \left(\hat{\Gamma}^{A}\right)\right] \\
F\left[\boldsymbol{\rho}^{B}\right]=\operatorname{Tr}\left[\hat{\Gamma}^{B} \hat{W}\right]+\frac{1}{\beta} \operatorname{Tr}\left[\hat{\Gamma}^{B} \ln \left(\hat{\Gamma}^{B}\right)\right]
\end{gathered}
$$

we obtain

$$
F[\boldsymbol{\rho}(\lambda)] \leq(1-\lambda) F\left[\boldsymbol{\rho}^{A}\right]+\lambda F\left[\boldsymbol{\rho}^{B}\right],
$$

which is equivalent to Eq. (A2). This concludes the proof that the density-matrix functional is convex.

\section{Appendix B: Invariance of the Kadanoff-Baym functional}

Here, we prove Eq. (21). We introduce

$$
\begin{aligned}
Y(\boldsymbol{\Delta}) & :=\Psi_{\beta, \mu}^{K B}[\boldsymbol{G}, \boldsymbol{\Sigma}+\boldsymbol{\Delta}, \boldsymbol{h}-\boldsymbol{\Delta}, \hat{W}] \\
& +\frac{1}{\beta} \sum_{\nu} \mathrm{e}^{i \beta \hbar \omega_{\nu} 0^{+}} \operatorname{Tr}[\boldsymbol{G} \boldsymbol{\Delta}]-\Psi_{\beta, \mu}^{K B}[\boldsymbol{G}, \boldsymbol{\Sigma}, \boldsymbol{h}, \hat{W}]
\end{aligned}
$$

which vanishes, when Eq. (21) is valid.

With Eq. (18), we obtain

$$
\begin{aligned}
Y(\boldsymbol{\Delta}) & =\frac{1}{\beta} \sum_{\nu} \mathrm{e}^{i \beta \hbar \omega_{\nu} 0^{+}} \operatorname{Tr} \ln \left(\frac{\left(i \hbar \omega_{\nu}+\mu\right) \mathbf{1}-\boldsymbol{h}+\boldsymbol{\Delta}}{\left(i \hbar \omega_{\nu}+\mu\right) \mathbf{1}-\boldsymbol{h}}\right) \\
& -\frac{1}{\beta} \operatorname{Tr} \ln \left(\frac{\mathbf{1}+\mathrm{e}^{-\beta(\boldsymbol{h}-\boldsymbol{\Delta}-\mu \mathbf{1})}}{\mathbf{1}+\mathrm{e}^{-\beta(\boldsymbol{h}-\mu \mathbf{1})}}\right)
\end{aligned}
$$

It is easily seen that $Y(\mathbf{0})=0$. In order to prove that $\boldsymbol{Y}(\boldsymbol{\Delta})=0$ for all arguments, we need to show that its derivative vanishes. We obtain

$$
\begin{aligned}
\frac{\partial Y}{\partial \boldsymbol{\Delta}} & =\frac{1}{\beta} \sum_{\nu} \mathrm{e}^{i \beta \hbar \omega_{\nu} 0^{+}} \frac{1}{\left(i \hbar \omega_{\nu}+\mu\right) \mathbf{1}-\boldsymbol{h}+\boldsymbol{\Delta}} \\
& -\frac{1}{1+\mathrm{e}^{\beta(\boldsymbol{h}-\boldsymbol{\Delta}-\mu \mathbf{1})}}=0
\end{aligned}
$$

which is verified using the elementary Matsubara sum ${ }^{29}$ $\frac{1}{\beta} \sum_{\nu} \mathrm{e}^{i \beta \hbar \omega_{\nu} 0^{+}} \frac{1}{i \hbar \omega_{\nu}-\epsilon}=\frac{1}{1+\mathrm{e}^{\beta \epsilon}}$. This concludes the proof.

\section{Appendix C: Functional derivative of the density-matrix functional}

Here, we show the explicit derivation of the derivative Eq. (35) of the density-matrix functional Eq. (30) and the minimum condition Eq. (36) for the grand potential

We begin with Eq. (30)

$$
\begin{aligned}
\tilde{F}_{\beta}^{\hat{W}}[\boldsymbol{\rho}] & =\frac{1}{\beta} \operatorname{Tr}[\boldsymbol{\rho} \ln (\boldsymbol{\rho})+(\mathbf{1}-\boldsymbol{\rho}) \ln (\mathbf{1}-\boldsymbol{\rho})] \\
& +\underset{\boldsymbol{h}^{\prime}}{\operatorname{stat} \operatorname{stat}}\left\{\Phi_{\beta}^{L W}[\boldsymbol{G}, \hat{W}]\right. \\
& -\frac{1}{\beta} \sum_{\nu} \operatorname{Tr}\left\{\ln \left[\mathbf{1}-\overline{\boldsymbol{G}}\left(\boldsymbol{h}^{\prime}+\boldsymbol{\Sigma}-\overline{\boldsymbol{h}}\right)\right]\right. \\
& \left.\left.+\left(\boldsymbol{h}^{\prime}+\boldsymbol{\Sigma}-\overline{\boldsymbol{h}}\right) \boldsymbol{G}-[\boldsymbol{G}-\overline{\boldsymbol{G}}]\left(\boldsymbol{h}^{\prime}-\overline{\boldsymbol{h}}\right)\right\}\right\}
\end{aligned}
$$

where we used $\overline{\boldsymbol{G}}\left(i \omega_{\nu}\right)$ defined earlier in Eq. (31).

We consider the dependencies via $\overline{\boldsymbol{h}}$ and $\overline{\boldsymbol{G}}$, while we exploit the stationary condition with respect to $\boldsymbol{G}, \boldsymbol{\Sigma}$ and $\boldsymbol{h}^{\prime}$.

$$
\begin{aligned}
& \frac{\partial \tilde{F}_{\beta}}{\partial \rho}=\frac{1}{\beta} \ln \left(\frac{\rho}{1-\boldsymbol{\rho}}\right) \\
& -\frac{1}{\beta} \sum_{\nu} \operatorname{Tr}\left\{\left[\mathbf{1}-\overline{\boldsymbol{G}}\left(\boldsymbol{h}^{\prime}+\boldsymbol{\Sigma}-\overline{\boldsymbol{h}}\right)\right]^{-1}\right. \\
& \times \underbrace{\left[-\overline{\boldsymbol{G}} \frac{\partial \overline{\boldsymbol{h}}}{\partial \boldsymbol{\rho}} \overline{\boldsymbol{G}}\left(\boldsymbol{h}^{\prime}+\boldsymbol{\Sigma}-\overline{\boldsymbol{h}}\right)+\overline{\boldsymbol{G}} \frac{\partial \overline{\boldsymbol{h}}}{\partial \boldsymbol{\rho}}\right]}_{\bar{G} \frac{\partial \bar{h}}{\partial \rho}\left[1-\bar{G}\left(\boldsymbol{h}^{\prime}+\boldsymbol{\Sigma}-\overline{\boldsymbol{h}}\right)\right]} \\
& \left.-\frac{\partial \overline{\boldsymbol{h}}}{\partial \boldsymbol{\rho}} \boldsymbol{G}+\frac{\partial \overline{\boldsymbol{G}}}{\partial \boldsymbol{\rho}}\left(\boldsymbol{h}^{\prime}-\overline{\boldsymbol{h}}\right)+[\boldsymbol{G}-\overline{\boldsymbol{G}}] \frac{\partial \overline{\boldsymbol{h}}}{\partial \boldsymbol{\rho}}\right\} \\
& =-\overline{\boldsymbol{h}}+\mu \mathbf{1}-\frac{1}{\beta} \sum_{\nu} \operatorname{Tr}\left\{\overline{\boldsymbol{G}} \frac{\partial \overline{\boldsymbol{h}}}{\partial \boldsymbol{\rho}}\right. \\
& \left.-\frac{\partial \overline{\boldsymbol{h}}}{\partial \boldsymbol{\rho}} \boldsymbol{G}+\frac{\partial \overline{\boldsymbol{G}}}{\partial \boldsymbol{\rho}}\left(\boldsymbol{h}^{\prime}-\overline{\boldsymbol{h}}\right)+[\boldsymbol{G}-\overline{\boldsymbol{G}}] \frac{\partial \overline{\boldsymbol{h}}}{\partial \boldsymbol{\rho}}\right\} \\
& =-\overline{\boldsymbol{h}}+\mu \mathbf{1}-\frac{1}{\beta} \sum_{\nu} \operatorname{Tr}\left\{\frac{\partial \overline{\boldsymbol{G}}}{\partial \boldsymbol{\rho}}\left(\boldsymbol{h}^{\prime}-\overline{\boldsymbol{h}}\right)\right\} \\
& =-\overline{\boldsymbol{h}}+\mu \mathbf{1}-\operatorname{Tr}\left\{\left(\boldsymbol{h}^{\prime}-\overline{\boldsymbol{h}}\right) \frac{\partial}{\partial \boldsymbol{\rho}}\left(\frac{1}{\beta} \sum_{\nu} \overline{\boldsymbol{G}}\right)\right\} \\
& =-\left(\boldsymbol{h}^{\prime}-\mu \mathbf{1}\right)
\end{aligned}
$$

This is the desired expression Eq. (35) for the derivative of the density-matrix functional.

Now, we turn to the stationary condition Eq. (36) for 
the grand potential

$$
\begin{aligned}
\Omega_{\beta, \mu}(\hat{h}+\hat{W}) & =\min _{\left|\psi_{n}\right\rangle, f_{n} \in[0,1]} \operatorname{stat}\left\{\sum_{n} f_{n}\left\langle\psi_{n}|\hat{h}| \psi_{n}\right\rangle\right. \\
& +F_{\beta}^{\hat{W}}\left[\sum_{n}\left|\psi_{n}\right\rangle f_{n}\left\langle\psi_{n}\right|\right]-\mu \sum_{n} f_{n} \\
& \left.-\sum_{m, n} \Lambda_{m, n}\left(\left\langle\psi_{n} \mid \psi_{m}\right\rangle-\delta_{n, m}\right)\right\}
\end{aligned}
$$

specified in Eq. (29).

The minimum conditions for Eq. (C3) with respect to the natural orbitals and occupations are

$$
\begin{array}{r}
\left(\hat{h}+\frac{\partial F_{\beta}^{\hat{W}}}{\partial \hat{\rho}}\right)\left|\psi_{n}\right\rangle-\sum_{m}\left|\psi_{m}\right\rangle \Lambda_{m, n} \frac{1}{f_{n}}=0 \\
\left\langle\psi_{n}\left|\hat{h}+\frac{\partial F_{\beta}^{\hat{W}}}{\partial \hat{\rho}}\right| \psi_{n}\right\rangle-\mu=0
\end{array}
$$

From the first equation, Eq. (C4), we obtain, exploiting orthonormality of the natural orbitals $\left|\psi_{n}\right\rangle$,

$$
\left\langle\psi_{m}\left|\left(\hat{h}+\frac{\partial F_{\beta}^{\hat{W}}}{\partial \hat{\rho}}\right)\right| \psi_{n}\right\rangle f_{n}=\Lambda_{m, n}
$$

From the variation with respect to the ket $\left\langle\psi_{n}\right|$, we obtain an equation analogous to Eq. (C6)

$$
f_{m}\left\langle\psi_{m}\left|\left(\hat{h}+\frac{\partial F_{\beta}^{\hat{W}}}{\partial \hat{\rho}}\right)\right| \psi_{n}\right\rangle=\Lambda_{m, n}
$$

The two equations Eq. (C6) and Eq. (C7) can be combined into

$$
\left\langle\psi_{m}\left|\left(\hat{h}+\frac{\partial F_{\beta}^{\hat{W}}}{\partial \hat{\rho}}\right)\right| \psi_{n}\right\rangle\left(f_{m}-f_{n}\right)=0
$$

As long as the occupations are all different, Eqs. (C8) and Eq. (C5) can be combined into the condition

$$
\frac{\partial F_{\beta}^{\hat{W}}}{\partial \hat{\rho}}=-\hat{h}+\mu \hat{1}
$$

Combining Eq. (C2) and Eq. (C9), the minimum condition for Eq. (C3) with respect to natural orbitals and occupations has the form

$$
\hat{h}=\hat{h}^{\prime}
$$

where $\boldsymbol{h}$ is the external potential and kinetic energy, while $\boldsymbol{h}^{\prime}$ is part of the Lagrange multiplier from the densitymatrix constraint in the density-matrix functional.

\section{Appendix D: Adiabatic connection at finite temperature}

The grand potential in density-functional theory is

$$
\Omega_{\beta, \mu}\left[v_{\text {ext }}\right]=\min _{n(\vec{r})}\left\{G_{\beta}^{\hat{W}}[n]+\int d^{3} r n(\vec{r}) v_{\text {ext }}(\vec{r})\right\}
$$

where $G_{\beta}^{\lambda \hat{W}}[n]$ is the universal density functional for a $\lambda$-scaled interaction $\lambda \hat{W}$

$$
\begin{aligned}
G_{\beta}^{\lambda \hat{W}}[n] & :=\min _{\left\{\left|\Phi_{j}\right\rangle, P_{j}\right\} \in M[n]}\left\{\sum_{j} P_{j}\left\langle\Phi_{j}|\hat{T}+\lambda \hat{W}| \Phi_{j}\right\rangle\right. \\
& \left.+\frac{1}{\beta} \sum_{j} P_{j} \ln \left(P_{j}\right)\right\} \\
& =\sum_{j} \bar{P}_{j}(\lambda)\left\langle\bar{\Phi}_{j}(\lambda)|\hat{T}+\lambda \hat{W}| \bar{\Phi}_{j}(\lambda)\right\rangle \\
& +\frac{1}{\beta} \sum_{j} \bar{P}_{j}(\lambda) \ln \left(\bar{P}_{j}(\lambda)\right)
\end{aligned}
$$

where $\hat{T}$ is the kinetic energy operator. In the first line, $M[n]$ is the set of all fermionic many-particle ensembles that produce the density $n(\vec{r})$. The many-particle ensembles are characterized by antisymmetric and orthonormal many-particle wave functions and non-negative probabilities that add up to one. The $\lambda$-dependent quantities $\left|\bar{\Phi}_{j}(\lambda)\right\rangle$ and $\bar{P}_{j}(\lambda)$ are those obtained by satisfying the minimum conditions with the scaled interaction $\lambda \hat{W}$.

Now, we follow the ensemble for a given density adiabatically from the non-interacting to the fully interacting case. The functional at full interaction is obtained as interaction-strength integral of the derivative. The latter can be simplified by exploiting the Hellmann-Feynman theorem, that is, by exploiting that the derivatives with respect to wave functions and probabilities vanish.

$$
\begin{aligned}
G_{\beta}^{\hat{W}}[n] & =G_{\beta}^{\hat{0}}[n]+\int_{0}^{1} d \lambda \frac{d G_{\beta}^{\lambda \hat{W}}[n]}{d \lambda} \\
& =K_{\beta}[n]+\int_{0}^{1} d \lambda \sum_{j} \bar{P}_{j}(\lambda)\left\langle\bar{\Phi}_{j}(\lambda)|\hat{W}| \bar{\Phi}_{j}(\lambda)\right\rangle \\
& =K_{\beta}[n]+E_{H}[n]+\int_{0}^{1} d \lambda U_{x c}^{\lambda, \beta}[n]
\end{aligned}
$$

The intrinsic energy $K_{\beta}[n]$ of non-interacting electrons is calculated as a minimization over Kohn-Sham wave functions $\left|\psi_{n}^{K S}\right\rangle$ and their occupations $f_{n}^{K S}$.

$$
\begin{aligned}
K_{\beta}[n] & =\min _{f_{n}^{K S},\left|\psi_{n}^{K S}\right\rangle v_{e f f}, \Lambda} \operatorname{stat}_{n} f_{n}^{K S}\left\langle\psi_{n}^{K S}\left|\frac{\hat{\vec{p}}^{2}}{2 m_{e}}\right| \psi_{n}^{K S}\right\rangle \\
& +\frac{1}{\beta} \sum_{n}\left[f_{n}^{K S} \ln \left(f_{n}^{K S}\right)+\left(1-f_{n}^{K S}\right) \ln \left(1-f_{n}^{K S}\right)\right] \\
& +\int d^{3} r v_{e f f}(\vec{r})\left[\sum_{n} f_{n}^{K S}\left|\psi_{n}^{K S}(\vec{r})\right|^{2}-n(\vec{r})\right] \\
& -\sum_{m, n} \Lambda_{m, n}\left(\left\langle\psi_{n}^{K S} \mid \psi_{m}^{K S}\right\rangle-\delta_{n, m}\right)
\end{aligned}
$$

The effective potential $v_{e f f}(\vec{r})$ is, like $\boldsymbol{\Lambda}$, a Lagrange multiplier.

$E_{H}$ is the Hartree energy

$$
E_{H}[n]:=\frac{1}{2} \int d^{3} r \int d^{3} r^{\prime} \frac{e^{2} n(\vec{r}) n\left(\overrightarrow{r^{\prime}}\right)}{4 \pi \epsilon_{0}\left|\vec{r}-\overrightarrow{r^{\prime}}\right|}
$$


and

$$
U_{x c}^{\lambda, \beta}[n]=\int d^{3} r n(\vec{r})\left\{\frac{1}{2} \int d^{3} r^{\prime} \frac{e^{2} h_{\beta, \lambda}\left(\vec{r}, \overrightarrow{r^{\prime}}\right)}{4 \pi \epsilon_{0}\left|\vec{r}-\overrightarrow{r^{\prime}}\right|}\right\}
$$

is the electrostatic interaction energy of the electrons with their respective exchange-correlation hole $h_{\beta, \lambda}\left(\vec{r}, \overrightarrow{r^{\prime}}\right)$. The latter is obtained at the specified temperature and scaled interaction strength. The hole function $h_{\beta, \lambda}\left(\vec{r}, \overrightarrow{r^{\prime}}\right)$ is related to the two-particle density $n^{(2)}\left(\vec{r}, \overrightarrow{r^{\prime}}\right)$ $\operatorname{via} n^{(2)}\left(\vec{r}, \overrightarrow{r^{\prime}}\right)=n(\vec{r})\left(n\left(\overrightarrow{r^{\prime}}\right)+h_{\beta, \lambda}\left(\vec{r}, \overrightarrow{r^{\prime}}\right)\right)$. Without interaction, Eq. (D6) provides the exchange energy $U_{x c}^{0, \beta}[n]$.
Thus, the exchange correlation energy $E_{x c}[n]$ can be constructed, like in the zero-temperature limit, as an interaction-strength average of a purely electrostatic term.

$$
E_{x c}[n]=\int_{0}^{1} d \lambda U_{x c, \beta}^{\lambda}[n]
$$

The exchange correlation hole, however, is temperature dependent. Via the Hellmann-Feynman theorem, it contains contributions from kinetic energy and entropy term. The main contribution to kinetic and entropy term is contained in $K_{\beta}[n]$ which considers both contributions of the non-interacting electron gas.
* corresponding author: peter.bloechl@tu-clausthal.de

1 P. Hohenberg and W. Kohn, Phys. Rev. 136, B864 (1964).

2 W. Kohn and L. J. Sham, Phys. Rev. 140, A1133 (1965).

3 M. Levy, Proc. Nat'l Acad. Sci. USA 76, 6062 (1979).

${ }^{4}$ R. M. Dreizler and E. K. U. Gross, Density Functional Theory (Springer, Berlin, 1990).

5 T. L. Gilbert, Phys. Rev. B 12, 2111 (1975).

6 A. Coleman, Rev. Mod. Phys. 35, 668 (1963).

7 A. M. K. Müller, Phys. Lett. 105, 446 (1984).

8 E. J. Baerends, Phys. Rev. Lett 87, 133004 (2001).

9 S. Sharma, J. K. Dewhurst, N. N. Lathiotakis, and E. K. U. Gross, Phys. Rev. B 78, 201103 (2008).

10 N. N. Lathiotakis, S. Sharma, J. K. Dewhurst, F. G. Eich, M. A. L. Marques, and E. K. U. Gross, Phys. Rev. A 79, 040501 (2009).

11 N. Mermin, Physical Review 137, A1441 (1965).

12 J. M. Luttinger and J. C. Ward, Phys. Rev. 118, 1417 (1960).

13 G. Baym and L. P. Kadanoff, Phys. Rev. 124, 287 (1961).

14 G. Baym, Phys. Rev. 127, 1391 (1962).

15 R. Chitra and G. Kotliar, Phys. Rev. B 62, 12715 (2000).

16 M. Potthoff, Eur. Phys. J. B 32, 429 (2003).

17 A. Georges, AIP Conf. Proc. 715, 3 (2004).

18 M. Potthoff, Condens. Matter Phys. 9, 557 (2006).

19 R. Chitra and G. Kotliar, Phys. Rev. B 63, 115110 (2001).

20 A. H. Nevidomskyy, D. Sénéchal, and A.-M. S. Tremblay, Phys. Rev. B 77, 075105 (2008).

21 G. Kotliar, Eur. Phys. J. B 11, 27 (1999).

22 C.-O. Almbladh, U. von Barth, and R. van Leeuwen, Int. J. Mod. Phys. B 13, 535 (1999).

23 N. E. Dahlen and U. vonBarth, Phys. Rev. B 69, 195102 (2004).

24 N. E. Dahlen, R. van Leeuwen, and U. von Barth, Int. J. Quant. Chem. 101, 512 (2005).

25 N. E. Dahlen, R. van Leeuwen, and U. von Barth, Phys. Rev. A 73, 012511 (2006).

26 W. H. Press, S. A. Teukolsky, W. T. Vetterling, and B. P. Flannery, Numerical Recipes, 3rd ed. (Cambridge University Press, New York, 2007).

27 R. Car and M. Parrinello, Phys. Rev. Lett 55, 2471 (1985).

28 P.-O. Löwdin, Phys. Rev. 97, 1474 (1955).

29 A. Fetter and J. Walecka,
Quantum Theory of Many-Particle Systems Hill, New York, 1971).

30 A. A. Abrikosow, L. P. Gorkov, and I. E. Dzyaloshinski, Methods of Quantum Field Theory in Statistical Physics (Prentice-Hall, Englewood Cliffs, NJ, 1964).

31 F. Lechermann, A. Georges, A. Poteryaev, S. Biermann, M. Posternak, A. Yamasaki, and O. K. Andersen, Phys. Rev. B 74, 125120 (2006).

32 M. Aichhorn, L. Pourovskii, V. Vildosola, M. Ferrero, O. Parcollet, T. Miyake, A. Georges, and S. Biermann, Phys. Rev. B 80, 085101 (2009).

${ }^{33}$ K. Haule, C.-H. Yee, and K. Kim, Phys. Rev. B 81, 195107 (2010).

34 B. Amadon, J. Phys.: Condens. Matter 24, 075604 (2012).

35 J. Harris, Phys. Rev. A 29, 1648 (1984).

36 V. I. Anisimov, J. Zaanen, and O. K. Andersen, Phys. Rev. B 44, 943 (1991).

37 A. I. Liechtenstein, V. I. Anisimov, and J. Zaanen, Phys. Rev. B 52, R5467 (1995).

38 A. D. Becke, J. Chem. Phys. 98, 1372 (1993).

39 A. D. Becke, J. Chem. Phys 98, 5648 (1993).

40 P. E. Blöchl, C. F. J. Walther, and T. Pruschke, Phys. Rev. B 84, 205101 (2011).

41 L. Hedin, Phys. Rev. 139, A796 (1965).

42 N. E. Bickers and D. Scalapino, Ann. Phys. 193, 206 (1989).

43 C. de Dominicis and P. C. Martin, J. Math. Phys. 5, 14 (1964).

44 A. Georges, G. Kotliar, W. Krauth, and M. J. Rozenberg, Rev. Mod. Phys. 68, 13 (1996).

${ }^{45} \mathrm{~W}$ Metzner and D. Vollhardt, Phys. Rev. Lett. 62, 324 (1989).

46 E. Müller-Hartmann, Z. Phys. B 74, 507 (1989).

47 P. Pulay, Mol. Phys. 17, 197 (1969).

48 A. Mackintosh and O. Andersen, "Electrons at the fermi surface of transition metals," (Cambridge University Press, New York, 1980) Chap. The electronic structure of transition metals, p. 149.

49 T. Koopmans, Physica 1, 104 (1934).

50 A. Szabo and N. S. Ostlund, Modern Quantum Chemistry (McGraw-Hill, New York, 1989).

51 A. Wehrl, Rev. Mod. Phys. 50, 221 (1978). 